Check for updates

Cite this: RSC Adv., 2018, 8, 17471

\title{
Europium ion post-functionalized zirconium metal-organic frameworks as luminescent probes for effectively sensing hydrazine hydrate $\uparrow$
}

\author{
Yunhui Yang, ${ }^{a}$ Xiaofei Liu, ${ }^{a}$ Dan Yan, ${ }^{b}$ Ping Deng, (D) a Zhiyong Guo (D) *a \\ and Hongbing Zhan ${ }^{\text {ac }}$
}

We describe a highly sensitive chemical sensor for the detection of the hydrazine hydrate. The chemical sensor was synthesized by simply doping a UiO-66 type metal-organic framework (MOF) with $\mathrm{Eu}^{3+}$ through a well-known post-synthetic modification method. The $\mathrm{Eu}^{3+}$ aMOF was characterized by powder X-ray diffraction (PXRD), nitrogen gas adsorption isotherm measurements, FTIR, and ICP-MS. This luminescent probe exhibits strong emission intensity, and more significantly, displays various merits, such as high selectivity with notable photoluminescence quenching effect, rapid response time, and remarkable sensitivity with low detection of limit for hydrazine recognition. Under the optimal experimental conditions, hydrazine hydrate can be detected by this method in concentrations as low as $0.18 \mu \mathrm{M}$, which is much lower than the threshold limit value $(10 \mathrm{ppb}, \approx 0.3 \mu \mathrm{M})$ of hydrazine exposure recommended by the U.S. Environmental Protection Agency (EPA). More interestingly, a portable film sensing device derived from this MOF display quick response to hydrazine hydrate within $90 \mathrm{~s}$. A possible luminescent quenching mechanism for hydrazine hydrate was also investigated.

Received 9th April 2018
Accepted 4th May 2018

DOI: $10.1039 / c 8 r a 03049 a$

rsc.li/rsc-advances

limit value (TLV) of $10 \mathrm{ppb}$ was recommended by the U.S.

\section{Introduction}

Hydrazine $\left(\mathrm{N}_{2} \mathrm{H}_{4}\right)$, as a highly reactive base and strong reducing agent, is widely used in medicine, pesticides, corrosion inhibitors, pharmaceutical intermediates and textile dyes. ${ }^{1-6}$ Due to its high enthalpy of combustion, hydrazine can also be used as a propellant in missile and rocket propulsion systems. ${ }^{7,8}$ Unluckily, this kind of chemical is very toxic and dangerously unstable especially in the anhydrous state. The widespread utilization of hydrazine gives rise to a high risk of explosion which may bring about a significant environmental disaster and even seriously casualty accident. Besides, due to its high volatility at room temperature, hydrazine can be readily absorbed and accumulated in organic organisms through skin contact and oral or even inhalation. Trace amount of hydrazine absorbed in human body can induce temporary blindness, irritation of nose, pulmonary enema, kidney damage, and undermine central nervous system..$^{9-12}$ On account of the considerable toxicological effects, hydrazine has been listed as one of the possible carcinogenic substances and its exposure threshold

${ }^{a}$ College of Materials Science and Engineering, Fuzhou University, Fuzhou, Fujian, 350108, PR China. E-mail: guozhy@fzu.edu.cn

${ }^{b}$ Testing Center, Fuzhou University, Fuzhou, Fujian, PR China

${ }^{c}$ Key Laboratory of Eco-materials Advanced Technology, Fuzhou University, Fujian Province, PR China

$\dagger$ Electronic supplementary information (ESI) available. See DOI: 10.1039/c8ra03049a
Environmental Protection Agency (EPA). ${ }^{13}$ Therefore, it is necessary to focus on the development of reliable, sensitive and convenient methods for the detection of hydrazine.

Many conventional analytical approaches have been employed to detect trace amounts of hydrazine, which include gas chromatography, ${ }^{14,15}$ HPLC, ${ }^{16,17}$ capillary electrophoresis, ${ }^{18}$ titrimetry $^{\mathbf{1 9}}$ and electrochemical techniques. ${ }^{\mathbf{2 0}-22}$ Among them, electrochemical analysis has been considered as a fast, sensitive and precise methodology with low limit of detection for hydrazine recognition. However, this kind of technology usually possesses some drawbacks. For instance, the surface of the conventional working electrodes such as the glassy carbon electrode (GCE) could suffer a sluggish kinetics because of the contact of hydrazine. Therefore, tedious and sophisticated processes of modifying electrodes would be needed, which may be time-consuming and high expenses. Other chromogenic chemosensors for hydrazine sensing also present some limitations, such as expensive experimental equipment, long analysis time (overnight), which inevitably restrict their practical application. In recent years, luminescence sensors have been widely developed and gradually become a prominent way for effective hydrazine sensing, primarily because of their facility, low cost, relatively rapid response, high sensitivity and selectivity. To date, owing to their unique recognition sites, only a limited number of luminescent probes that based on organic molecules or polymers have been studied for detecting hydrazine. Nevertheless, complicated synthetic routines and various toxic 
solvents used during the preparation of luminescent probes may contaminate the environment and damage the living organisms. Additionally, a few probes could be only available under some specific conditions, such as in acidic atmosphere. ${ }^{23-25}$ Therefore, it is strongly urgent to explore proper luminescent materials for online hydrazine monitoring in a simple, economical and environment-friendly way. As an emerging class of crystalline materials, luminescent lanthanide-organic frameworks (Ln-MOFs) have drawn extensive attention due to their perfect combination of tuneable and regular structures of MOFs and unique optical properties of $\mathrm{Ln}^{3+}$ through an "antenna effect". ${ }^{26-28}$ Up to now, Ln-MOFs have been used as the excellent and promising candidates for luminescent probes to detect metal ions, ${ }^{29}$ anions, ${ }^{30}$ organic small molecules, ${ }^{31,32}$ proteins,${ }^{33}$ toxic gases. ${ }^{34}$ However, to the best of our knowledge, luminescent Ln-MOFs based sensors for hydrazine detection have not been reported yet.

In this contribution, a lanthanide functionalized luminescent probe $\mathrm{Eu}^{3+}$ @UiO-66- $(\mathrm{COOH})_{2}$ is prepared to qualitative and quantitative analysis of hydrazine. The utilization of this Eu-doped UiO-66 analogue for sensing hydrazine is considered to be the following two reasons: (i) UiO-66 analogue has an excellent chemical stability, which can maintain its structure even when encountering in a stronger base atmosphere $(\mathrm{pH} \approx$ 14); ${ }^{35}$ (ii) the introduction of $\mathrm{Eu}^{3+}$ can be efficiently sensitized based on linker-to-metal energy transfer (LMET), and this process would be easily hampered when specific analyte is introduced to the system, resulting in a luminescence quenching effect. Therefore, the alkaline property of hydrazine is an ideal analyte and could be expected to interact with the framework. This Ln-MOF exhibits an excellent performance with high sensitivity, selectivity, and rapid response for hydrazine detection. Furthermore, this luminescent probe can be employed in both solution and vapour states.

\section{Experimental section}

\section{Materials and reagents}

All the reagents and solvents were purchased from commercial sources and used without further purification. Europium nitrate hexahydrate $\left(\mathrm{Eu}\left(\mathrm{NO}_{3}\right)_{3} \cdot 6 \mathrm{H}_{2} \mathrm{O}\right)$ was purchased from Jiangsu Guosheng Co. Ltd. (Jiangsu, China). 1,2,4,5-Benzenetetracarboxylic acid $\left(\mathrm{H}_{4}\right.$ btec, $\left.>80 \%\right)$, zirconium tetrachloride $\left(\mathrm{ZrCl}_{4}, 98 \%\right)$, trimethylamine (TEA, $\left.\geq 99.0 \%\right)$, hydrazine hydrate $(>98.0 \%)$ were purchased from Aladdin Ltd. (Shanghai, China). $N, N^{\prime}$-dimethylformamide (DMF, 99.5\%), acetone ( $\left.\geq 99.5 \%\right)$ were purchased from Sinopharm Chemical Reagent Co. Ltd. (Shanghai, China) and were reagent grade. Deionizedultrafiltered (>18.25 $\mathrm{M} \Omega \mathrm{cm}$ ) water was used to prepare all aqueous solutions.

\section{Apparatus and measurements}

The powder X-ray diffraction (PXRD) data were collected on a Miniflex 600 diffractometer at room temperature using $\mathrm{Cu} \mathrm{K} \alpha$ radiation $(\lambda=0.15418 \mathrm{~nm})$ with $15 \mathrm{~mA}, 40 \mathrm{kV}$, and a scan rate of $3^{\circ} \min ^{-1}\left(2 \theta, 3-40^{\circ}\right)$. The Fourier transform infrared (FT-IR) spectra were recorded in the wavenumber range of 4000$400 \mathrm{~cm}^{-1}$ by utilizing $\mathrm{KBr}$ pellets pressing method on a Thermo Fisher Nicolet 5700 spectrometer. ICP-MS was performed on Thermo Scientific XSERIES 2 inductively coupled plasma-mass spectrometry (ICP-MS) system. Nitrogen adsorption/ desorption isotherms were tested at liquid nitrogen temperature using a Micromeritics ASAP 2460 surface area analyser. Prior to sorption measurements, the $\mathrm{Eu}^{3+}$ @UiO-66- $(\mathrm{COOH})_{2}$ was activated at $80{ }^{\circ} \mathrm{C}$ overnight. Surface areas of the products were calculated using the Brunauer-Emmett-Teller (BET) method. ICP-MS data were collected on a Thermo Scientific XSERIES 2 inductively coupled plasma-mass spectrometer. Luminescence excitation and emission spectra were conducted on a FluoroMax-4 spectrofluorometer at room temperature utilizing a $450 \mathrm{~W}$ xenon lamp as excitation source, and luminescence lifetimes $(\tau)$ measurements were taken with an Edinburgh FLS980 spectrophotometer.

\section{Detailed description of the experiment}

Synthesis of Eu ${ }^{3+}$ @UiO-66-(COOH $)_{2}$. The UiO-66- $(\mathrm{COOH})_{2}$ solid sample was synthesized according to the previously described procedure. ${ }^{36}$ A mixture of $0.1 \mathrm{~g}$ UiO-66-(COOH $)_{2}$ and $0.0366 \mathrm{~g} \mathrm{Eu}\left(\mathrm{NO}_{3}\right)_{3} \cdot 6 \mathrm{H}_{2} \mathrm{O}$ was added to round-bottom flask, and then uniformly dispersed in $10 \mathrm{~mL}$ distilled water. After ultrasonic agitation for 10 minutes, the mixture was stirring and heated at $60{ }^{\circ} \mathrm{C}$ for $24 \mathrm{~h}$. The solid was then filtered off, extensively washed with distilled water and dried under vacuum at $60{ }^{\circ} \mathrm{C}$ overnight, resulting in a white power sample.

Fabrication of luminescent film of $\mathrm{Eu}^{3+}$ @UiO-66-(COOH $)_{2}$. The solid sample of $\mathrm{Eu}^{3+}$ @UiO-66- $(\mathrm{COOH})_{2}$ was dispersed in ethanol and ultrasonicated for $10 \mathrm{~min}$. Before film coating, the substrates were immersed in ethanol and rinsed for several times. Drop-casting method was used during the fabrication of the film. The suspension-state sample was carefully dropping onto the substrate, and this process was repeated several times. Then, the obtained film was dried at room temperature.

Photoluminescence (PL) sensing experiments. $\mathrm{Eu}^{3+} @ \mathrm{UiO}-$ $66-(\mathrm{COOH})_{2}(2 \mathrm{mg})$ was crushed to obtain a fine powder and was uniformly dispersed in $1 \mathrm{~mL}$ ethanol. The formed suspension was sonicated for about 10 minutes followed by adding different amount of hydrazine hydrate. After standing for a while, the suspension was transferred into the fluorescence cuvette for the photoluminescence studies. The excitation and emission spectra were collected at room temperature, and the slit widths of excitation and emission remain the same in every luminescent measurement.

\section{Results and discussion}

\section{Crystal structure and characterization}

In recent years, due to the excellent chemical and thermal stability, UiO-66 analogues have aroused a great deal of research. Among them, UiO-66- $(\mathrm{COOH})_{2}$ is an attractive material in which two carboxylic acid groups are left protonated. ${ }^{37}$ By means of introducing the targeted $\mathrm{Eu}^{3+}$ into pores of the structure via a simple post-synthetic modification, the 
lanthanide-organic framework $\mathrm{Eu}^{3+} @ U i \mathrm{O}-66-(\mathrm{COOH})_{2}$ was synthesized (Scheme 1). As shown in the infrared spectroscopy (ESI, Fig. S1 $\dagger$ ), the band observed at $1715 \mathrm{~cm}^{-1}$ is attributed to the vibration of the uncoordinated carboxylate groups become weaker, suggesting $\mathrm{Eu}^{3+}$ is successfully coordinated with the free carboxyl in the framework. Meanwhile, PXRD pattern (ESI, Fig. S2 $\dagger)$ of the $\mathrm{Eu}^{3+}$ @UiO-66- $(\mathrm{COOH})_{2}$ is almost unchanged compared to that of the UiO-66-(COOH $)_{2}$, which indicates that the incorporation of $\mathrm{Eu}^{3+}$ does not affect the stability and crystalline integrity of the framework. The permanent porosity of $\mathrm{Eu}^{3+}$ @UiO-66-(COOH $)_{2}$ was confirmed by the $\mathrm{N}_{2}$ adsorptiondesorption isotherm. The BET surface area of Eu ${ }^{3+}$ @UiO-66$(\mathrm{COOH})_{2}$ is about $181 \mathrm{~m}^{2} \mathrm{~g}^{-1}$ (ESI, Fig. S3†), which is lower than that of UiO-66-(COOH $)_{2}$. The element value of $\mathrm{Zr}^{4+}$ and $\mathrm{Eu}^{3+}$ in $\mathrm{Eu}^{3+} @$ UiO-66- $(\mathrm{COOH})_{2}$ was quantified by ICP-MS measurement (ESI, Table $\mathrm{S} 1 \dagger$ ), and the result illustrates the molar ratio of $\mathrm{Zr}^{4+}$ and $\mathrm{Eu}^{3+}$ is about 2 .

\section{Photoluminescence properties}

The successful incorporation of $\mathrm{Eu}^{3+}$ into UiO-66-(COOH $)_{2}$ encourages us to explore its luminescent property. As shown in Fig. $\mathrm{S} 4, \uparrow$ the luminescent emission spectrum exhibits sharp characteristic peaks of $\mathrm{Eu}^{3+}$, while the emission band of the framework is remarkably suppressed. In general, the sharp and narrow emission peaks appearing at 578, 590, 611, 650 and $698 \mathrm{~nm}$ could be ascribed to ${ }^{5} \mathrm{D}_{0} \rightarrow{ }^{7} \mathrm{~F}_{j}(j=0,1,2,3,4)$ transitions of the $\mathrm{Eu}^{3+}$ ions, respectively. Among these emission bands, the highest intensity is located at $611 \mathrm{~nm}$. In the existence of energy transfer from $\mathrm{H}_{4}$ btec ligands to $\mathrm{Eu}^{3+}$ ions through "antenna effect", the luminescence of $\mathrm{Eu}^{3+}$ in the framework is sensitized, which is implied by the excitation spectrum of $\mathrm{Eu}^{3+} @ U i O-66-(\mathrm{COOH})_{2}($ monitored at $611 \mathrm{~nm})$. The excellent luminescent property and its innate good chemical stability make $\mathrm{Eu}^{3+}$ @UiO-66- $(\mathrm{COOH})_{2}$ an ideal candidate for luminescent sensors, especially for detecting toxic substances in liquid systems.

\section{Sensing properties}

To determine whether Eu ${ }^{3+} @ U i O-66-(\mathrm{COOH})_{2}$ could be a highly sensitive luminescent probe for hydrazine detection, different kinds of N-containing compounds and organic bases such as urea, DMF, acetonitrile, pyridine, trimethylamine (TEA), ammonia have been introduced to the system under the same conditions. The suspension-state luminescent measurements

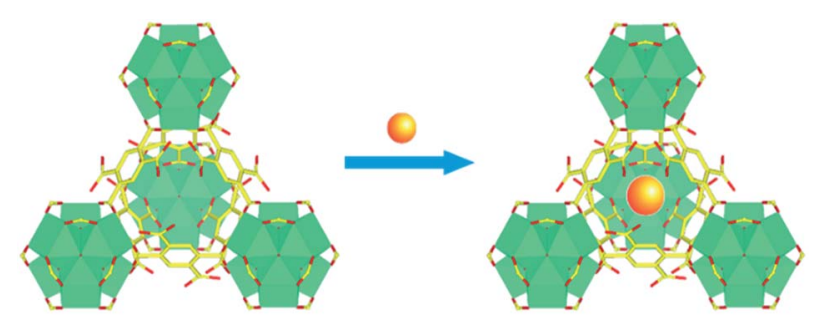

Scheme 1 Synthetic scheme of $\mathrm{Eu}^{3+} \mathrm{aUiO}-66-(\mathrm{COOH})_{2}$ with representative tetrahedral cage structure. illustrate that the addition of these components has quite different impacts on the luminescence intensity (Fig. 1a). As shown in Fig. 1b, when in contact with urea, DMF, pyridine, toluene and TEA, the luminescence intensity of Eu ${ }^{3+} @ U i O-66-$ $(\mathrm{COOH})_{2}$ changed slightly. The luminescence intensity of $\mathrm{Eu}^{3+}$ @UiO-66-(COOH $)_{2}$ was dropped about 40\% after adding the same amount of ammonia, which indicates a weak luminescent quenching kinetics. In contrast to other analytes, after adding hydrazine about 93\% luminescence intensity was dramatically suppressed, which evidently shows its highly selective luminescent quenching behaviour towards hydrazine. The quenching effect was also verified by the photograph obtained under a UV lamp irradiation, and only the hydrazine treated sample showed a visible colour change from bright red to the nearly nonluminous. The direct and simple way of visual perception by naked eyes suggests that $\mathrm{Eu}^{3+}$ @UiO-66- $(\mathrm{COOH})_{2}$ can act as a luminescent probe for highly selective detection of hydrazine (ESI, Fig. S5†).

Sensitivity is also a crucial factor to estimate the sensing performance of $\mathrm{Eu}^{3+}$ @UiO-66- $(\mathrm{COOH})_{2}$. As illustrated in Fig. 2a, when the hydrazine hydrate concentration increased from 0 to $50 \mathrm{mM}$, the emission intensity of Eu ${ }^{3+} @ U i O-66-(\mathrm{COOH})_{2}$ was gradually quenched. After adding $10 \mathrm{mM}$ hydrazine hydrate, its bright red emission was almost disappeared under UV light. In order to further evaluate the quenching effect, the dependence of the luminescence intensity on the hydrazine concentration was plotted. Fig. $2 \mathrm{~b}$ shows that the emission intensity decreases rapidly at the beginning. In the range of 0 to $1 \mathrm{mM}$, the linear relationship between the quenching intensity and the hydrazine
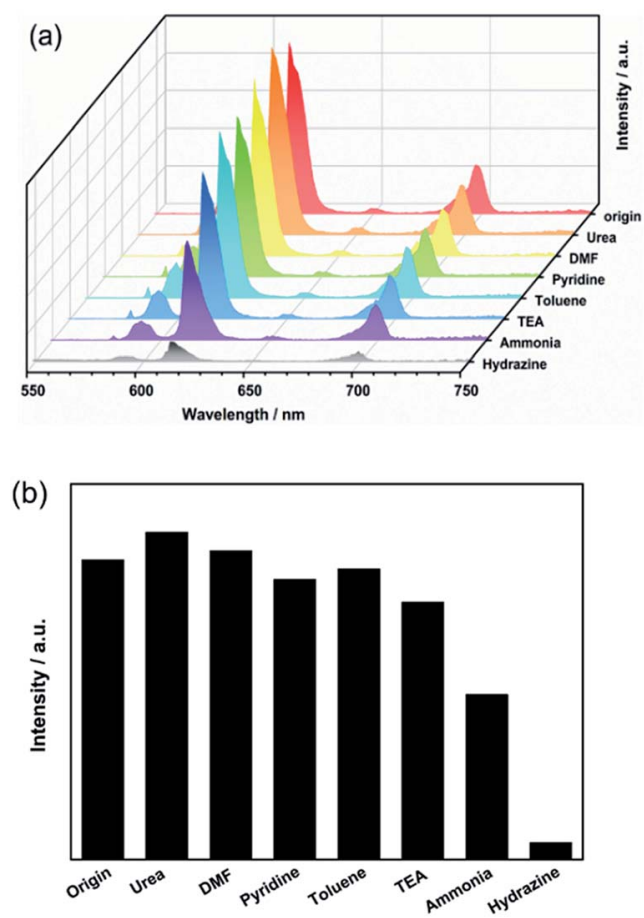

Fig. 1 (a) The luminescence spectra and (b) intensities (recorded at 611 $\mathrm{nm})$ of $\mathrm{Eu}^{3+} \mathrm{aUiO}-66-(\mathrm{COOH})_{2}$ suspensions towards various components $\left(\lambda_{\mathrm{ex}}=321 \mathrm{~nm}\right)$. 

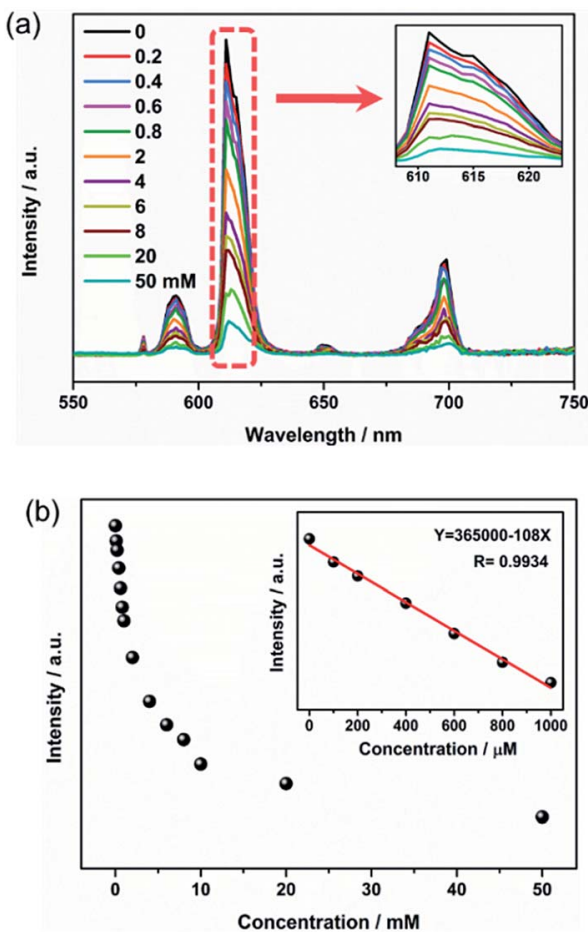

Fig. 2 (a) The $\mathrm{PL}$ emission of $\mathrm{Eu}^{3+} \mathrm{aUiO}-66-(\mathrm{COOH})_{2}$ under different concentrations of hydrazine. (b) Plot of luminescence intensity of $\mathrm{Eu}^{3+} \mathrm{aUiO}-66(\mathrm{COOH})_{2}$ at $611 \mathrm{~nm}$ under different hydrazine concentration.

concentration can be fitted as a function of eqn (1) with a correlation coefficient $\left(R^{2}\right)$ of 0.9934 .

$$
I=365000-108 C
$$

According to eqn (1), the detection of limit (LOD) for hydrazine is $0.18 \mu \mathrm{M}$, which is calculated from $3 \sigma / k$, where $k$ is the slope of the calibration curve and $\sigma$ is the standard for ten times replicating luminescence measurements of the blank.

The LOD is much lower than the threshold limit value (10 $\mathrm{ppb}, \approx 0.3 \mu \mathrm{M}$ ) of hydrazine exposure recommended by the U.S. Environmental Protection Agency (EPA).$^{13}$ In order to evaluate our sensing performance, we summarized most of the reported hydrazine detecting methods (Table 1 ). ${ }^{38-42}$ It should be noted that chromatography methods generally possess much lower detecting limit, though they are usually time-consuming and costly. In the aspect of LOD, our method is comparable to other detecting methods. Meanwhile, our method is more convenient than the electrochemical one and environment-friendly than the fluorescent dye one.

Besides, it is worth noting that the response rate of luminescence quenching after introducing hydrazine is very fast in our current system. When monitored the maximum emission intensity at $611 \mathrm{~nm}$, the time-dependent quenching efficiency curve (Fig. 3) showed a sharp decrease after addition of hydrazine within $30 \mathrm{~s}$ and the quenching efficiency was saturated within $1 \mathrm{~min}$. Hence, the results show this luminescent probe is highly sensitive and quick response to hydrazine. The above
Table 1 Comparison of the detection limit with different methods for sensing hydrazine

\begin{tabular}{|c|c|c|c|}
\hline Sensors & Methods & LOD & Ref. \\
\hline Naphthalene-2,3-dialdehyde & LC-MS/MS & $0.0006 \mu \mathrm{M}$ & 38 \\
\hline Naphthalene-2,3-dialdehyde & HPLC & $0.001 \mu \mathrm{M}$ & 39 \\
\hline $\mathrm{GO} / \mathrm{CTS} / \mathrm{Pt}$ & Electrochemistry & $3.6 \mu \mathrm{M}$ & 40 \\
\hline HAP-rGO-CS & Electrochemistry & $0.43 \mu \mathrm{M}$ & 41 \\
\hline & Luminescence & $0.25 \mu \mathrm{M}$ & 42 \\
\hline & Luminescence & $0.36 \mu \mathrm{M}$ & 24 \\
\hline $\mathrm{Eu}^{3+} @ \mathrm{UiO}-66-(\mathrm{COOH})_{2}$ & Luminescence & $0.18 \mu \mathrm{M}$ & This work \\
\hline
\end{tabular}

excellent performance of $\mathrm{Eu}^{3+} @ \mathrm{UiO}-66-(\mathrm{COOH})_{2}$ pave the way for its practical application in the field of chemical sensing.

The sensing outcome of $\mathrm{Eu}^{3+} @ \mathrm{UiO}-66-(\mathrm{COOH})_{2}$ towards hydrazine hydrate in solution prompts us to explore the luminescence quenching capability in the presence of hydrazine hydrate vapour. The film of Eu ${ }^{3+} @$ UiO-66- $(\mathrm{COOH})_{2}$ was formed on a quartz glass slide, and the fluorescence spectra were recorded before and after exposure to vapour phase of hydrazine hydrate over a period of given time interval. After $30 \mathrm{~s}$ of exposure to hydrazine hydrate vapour (ESI, Fig. S7, Video S1 $\dagger$ ), appreciable quenching in luminescence of $\mathrm{Eu}^{3+}$ @UiO-66$(\mathrm{COOH})_{2}$ has been observed. With the exposure time close to $90 \mathrm{~s}$, the emission of the film was almost disappeared. The difference of emission intensity under different hydrazine exposure time can be readily distinguished by the naked eye, indicating that $\mathrm{Eu}^{3+} @$ @UiO-66- $(\mathrm{COOH})_{2}$ can also be used for the real-time detection of hydrazine hydrate in the vapour phase.

\section{Possible mechanism of luminescent quenching}

In order to better understand the sensing process, we tentatively investigated the possible quenching mechanism of $\mathrm{Eu}^{3+} @ U$ UiO$66-(\mathrm{COOH})_{2}$. As for inorganic compounds, there are three

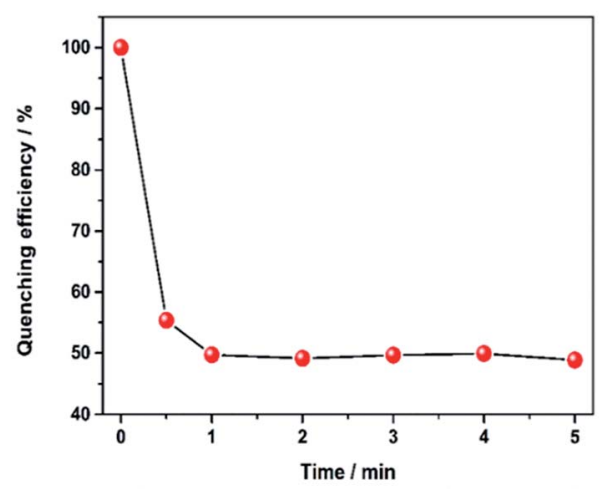

Fig. 3 Variation of the quenching efficiency at $611 \mathrm{~nm}$ as a function of immersion time in hydrazine $(2 \mathrm{mM})$. 


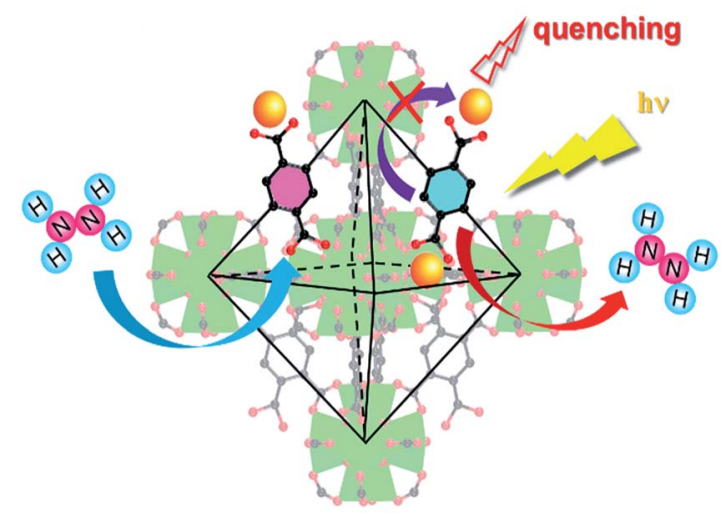

Fig. 4 Schematic view of the luminescent detection mechanism.

possible ways that could lead to the quenching effect of the host materials: (i) the introduction of the analysts causes the collapse of the framework; (ii) the direct interaction between the analytes and $\mathrm{Eu}^{3+}$ ions; (iii) the interaction of the organic linkers and the analytes, which would disorder the electric density of the linkers and further influence the process of energy transfer to $\mathrm{Eu}^{3+}$. As illustrated in Fig. S6, $\uparrow$ after the addition of hydrazine, the PXRD pattern is identical to that of the original one, suggesting the hydrazine does not affect the whole framework. According to the oxo-philic nature of early transition metal complexes, $\mathrm{Eu}^{3+}$ ions are more likely to coordinate with the oxygen donors instead of the nitrogen-rich compounds. Thus, the analyte hydrazine is less likely to interact with $\mathrm{Eu}^{3+}$, and this factor is further evidenced by the lifetime of luminescence and ICP-MS. After being treated with different concentrations of hydrazine, the luminescence lifetime of the $\mathrm{Eu}^{3+}$ @UiO-66- $(\mathrm{COOH})_{2}$ are almost unchanged (ESI, Fig. S8 $\dagger$ ). ICP-MS result suggests the molar ratio of $\mathrm{Zr}^{4+}$ and $\mathrm{Eu}^{3+}$ is maintained after contacting with hydrazine, indicating this analyte does not bring about $\mathrm{Eu}^{3+}$ leaching (ESI, Table S1†). Therefore, the luminescence quenching should be ascribed to the interactions between the hydrazine and the organic ligand $\mathrm{H}_{4}$ btec. Based on the fact that hydrazine is a kind of strong base, the carboxyl-rich ligand and hydrazine could react with each other, which is certified by the disappeared band of uncoordinated carboxyl at $1715 \mathrm{~cm}^{-1}$ in infrared spectroscopy (ESI, Fig. S1†). UV-vis spectra endow that the absorptive wavelength has a markedly difference in the presence of hydrazine, suggesting the interaction indeed exists between the ligand and analyte (ESI, Fig. S9†). As the concentration of hydrazine increased to $2 \mathrm{mM}$, the absorption peak had a hypsochromic shift from $216 \mathrm{~nm}$ to $206 \mathrm{~nm}$, revealing an increase in the energy level of the $\pi^{*}$ orbits of the ligands. ${ }^{43}$ As a result, the number of the excited photoelectrons in the framework is remarkably decreasing at a given energy, which in consequence weakens the ability of energy transfer from ligands to $\mathrm{Eu}^{3+}$ ions, resulting in a luminescence quenching effect (Fig. 4).

\section{Conclusions}

In the present work, we demonstrate a water stable lanthanide functionalized metal-organic framework $\mathrm{Eu}^{3+}$ @UiO-66-
$(\mathrm{COOH})_{2}$ as a luminescent probe to detect hydrazine hydrate. This luminescent probe shows an excellent selectivity among nitrogen-rich compounds and base reagents, high sensitivity with a low detection limit $(0.18 \mu \mathrm{M})$, and fast response time ( $\sim 30 \mathrm{~s}$ ) for sensing hydrazine hydrate in solution. Furthermore, a film device based on this luminescent probe was prepared in a simple, economical and portable way. The well performance of the film sensor (completely quenching within $90 \mathrm{~s}$ ) could motivate its practical application as a real-time monitor for online hydrazine detection. The sensing mechanism of hydrazine hydrate quenched luminescence is accredited to the energy transfer from ligand to analytes, which spontaneously decrease the energy transfer from the ligand to $\mathrm{Eu}^{3+}$.

\section{Conflicts of interest}

There are no conflicts to declare.

\section{Acknowledgements}

This work was supported by the National Natural Science Foundation of China (Grant No. 51703031 and 21704015).

\section{Notes and references}

1 S. D. Zelnick, D. R. Mattie and P. C. Stepaniak, Aviat., Space Environ. Med., 2003, 74, 1285-1291.

2 J. Fan, W. Sun, M. Hu, J. Cao, G. Cheng, H. Dong, K. Song, Y. Liu, S. Sun and X. Peng, Chem. Commun., 2012, 48, 8117-8119.

3 I. Cruz Vieira, K. Omuro Lupetti and O. Fatibello-Filho, Anal. Lett., 2002, 35, 2221-2231.

4 S. Zhu, W. Lin and L. Yuan, Anal. Methods, 2013, 5, 34503453.

5 L. Cui, C. Ji, Z. Peng, L. Zhong, C. Zhou, L. Yan, S. Qu, S. Zhang, C. Huang, X. Qian and Y. Xu, Anal. Chem., 2014, 86, 4611-4617.

6 A. Samadi-Maybodi, S. Ghasemi and H. Ghaffari-Rad, Sens. Actuators, B, 2015, 220, 627-633.

7 G. Wu, X. Tang, W. Ji, K. W. Lai and Q. Tong, Methods Appl. Fluoresc., 2017, 5, 015001.

8 X. Gu and J. P. Camden, Anal. Chem., 2015, 87, 6460-6464.

9 C. A. Reilly and S. D. Aust, Chem. Res. Toxicol., 1997, 10, 328334.

10 S. Garrod, M. E. Bollard, A. W. Nicholls, S. C. Connor, J. Connelly, J. K. Nicholson and E. Holmes, Chem. Res. Toxicol., 2005, 18, 115-122.

11 R. Maji, A. K. Mahapatra, K. Maiti, S. Mondal, S. S. Ali, P. Sahoo, S. Mandal, M. R. Uddin, S. Goswami, C. K. Quah and H. K. Fun, RSC Adv., 2016, 6, 70855-70862.

12 M. Matsumoto, H. Kano, M. Suzuki, T. Katagiri, Y. Umeda and S. Fukushima, Regul. Toxicol. Pharmacol., 2016, 76, 6373.

13 A. Umar, M. M. Rahman, S. H. Kim and Y.-B. Hahn, Chem. Commun., 2008, 2, 166-168.

14 W. E. Davis Ii and Y. Li, Anal. Chem., 2008, 80, 5449-5453. 
15 Y. Y. Liu, I. Schmeltz and D. Hoffmann, Anal. Chem., 1974, 46, 885-889.

16 G. Elias and W. F. Bauer, J. Sep. Sci., 2006, 29, 460-464.

17 J. A. Oh and H. S. Shin, J. Chromatogr. A, 2015, 1395, 73-78. 18 J. Liu, W. Zhou, T. You, F. Li, E. Wang and S. Dong, Anal. Chem., 1996, 68, 3350-3353.

19 Z. K. He, B. Fuhrmann and U. Spohn, Anal. Chim. Acta, 2000, 409, 83-91.

20 W. Hou and E. Wang, Anal. Chim. Acta, 1992, 257, 275-280.

21 S. V. Guerra, C. R. Xavier, S. Nakagaki and L. T. Kubota, Electroanalysis, 1998, 10, 462-466.

22 E. B. Shi, H. M. Lin, Q. Wang, F. Zhang, S. X. Shi, T. T. Zhang, X. Li, H. Niu and F. Y. Qu, Dalton Trans., 2017, 46, 554-563.

23 R. Y. Zhang, C. J. Zhang, Z. G. Song, J. Liang, R. T. K. Kwok, B. Tang and B. Liu, J. Mater. Chem. C, 2016, 4, 2834-2842.

24 X. Q. Zhang, C. L. Shi, P. W. Ji, X. D. Jin, J. N. Liu and H. J. Zhu, Anal. Methods, 2016, 8, 2267-2273.

25 S. W. Thomas and T. M. Swager, Adv. Mater., 2006, 18, 10471050.

26 Y. Cui, B. Li, H. He, W. Zhou, B. Chen and G. Qian, Acc. Chem. Res., 2016, 49, 483-493.

27 B. Li, H. M. Wen, Y. Cui, W. Zhou, G. Qian and B. Chen, Adv. Mater., 2016, 28, 8819-8860.

28 Y. J. Cui, Y. F. Yue, G. D. Qian and B. L. Chen, Chem. Rev., 2012, 112, 1126-1162.

29 Y. T. Liang, G. P. Yang, B. Liu, Y. T. Yan, Z.-P. Xi and Y.-Y. Wang, Dalton Trans., 2015, 44, 13325-13330.
30 X. Lian and B. Yan, Dalton Trans., 2016, 45, 18668-18675.

31 Z. Guo, X. Song, H. Lei, H. Wang, S. Su, H. Xu, G. Qian, H. Zhang and B. Chen, Chem. Commun., 2015, 51, 376-379.

32 Z. Guo, H. Xu, S. Su, J. Cai, S. Dang, S. Xiang, G. Qian, H. Zhang, M. O'Keeffe and B. Chen, Chem. Commun., 2011, 47, 5551-5553.

33 Y. Zhou, H. H. Chen and B. Yan, J. Mater. Chem. A, 2014, 2, 13691-13697.

34 J. Ma and B. Yan, J. Colloid Interface Sci., 2017, 513, 133-140. 35 Q. Yang, S. Vaesen, F. Ragon, A. D. Wiersum, D. Wu, A. Lago, T. Devic, C. Martineau, F. Taulelle, P. L. Llewellyn, H. Jobic, C. Zhong, C. Serre, G. De Weireld and G. Maurin, Angew. Chem., Int. Ed., 2013, 52, 10316-10320.

36 J. N. Hao and B. Yan, Chem. Commun., 2015, 51, 7737-7740. 37 X. Zhang, Q. Hu, T. F. Xia, J. Zhang, Y. Yang, Y. J. Cui, B. L. Chen and G. D. Qian, ACS Appl. Mater. Interfaces, 2016, 8, 32259-32265.

38 J. A. Oh and H.-S. Shin, J. Chromatogr. A, 2015, 1395, 73-78. 39 A. D. Smolenkov, A. V. Chernobrovkina, R. S. Smirnov and O. A. Shpigun, Anal. Chem., 2012, 67, 360-363.

40 D. Rao, Q. Sheng and J. Zheng, Sens. Actuators, B, 2016, 236, 192-200.

41 F. Gao, Q. Wang, N. Gao, Y. Yang, F. Cai, M. Yamane, F. Gao and H. Tanaka, Biosens. Bioelectron., 2017, 97, 238-245.

42 Y. H. Xiao, G. Xi, X. X. Zhao, S. Zhou, Z. Q. Zhou and B. X. Zhao, J. Fluoresc., 2015, 25, 1023.

43 J. N. Hao and B. Yan, Nanoscale, 2016, 8, 2881-2886. 